\title{
LA EVALUACIÓN DEL DESEMPEÑO PROFESIONAL DOCENTE: UNA MIRADA AL INSTITUTO SUPERIOR TECNOLÓGICO DE FORMACIÓN
}

Ing. Alejandro Lema Cachinell

Instituto Superior Tecnológico de Formación

alejandrol@formacion.edu.ec
Ing. Zulay Delgado Saeteros

Instituto Superior Tecnológico de Formación

zulayd@formacion.edu.ec

Recepción: 4 de enero de 2016

Aceptado: 13 de febrero de 2016
Palabras claves : Evaluación, proceso de desempeño profesional

Keywords: Evaluation, process of professional performance

\section{RESUMEN}

La presente investigación reflexiona entorno al proceso de evaluación y las insuficiencias en ese proceso que limitan el desempeño profesional de los docentes en los institutos superiores tecnológicos en Ecuador. Se realiza un breve análisis del proceso de evaluación en el contexto de los institutos tecnológicos de Ecuador y del desempeño profesional del docente. Se propone de igual manera un diagnóstico de la situación actual del Instituto Superior Tecnológico de Formación Profesional Administrativa y Comercial en relación con el proceso de evaluación del desempeño del docente.

\section{ABSTRACT}

This research reflects on the evaluation process and the shortcomings in this process that limit the professional performance of professors in the technological institutes in Ecuador. A brief analysis of the evaluation process is carried out in the context of the technological institutes of Ecuador and the professional performance of professors. It also proposes a diagnosis of the current situation of Instituto Superior Tecnológico de Formación Profesional Administrativa y Comercial in relation to the process of evaluation of the professor's performance. 


\section{INTRODUCCIÓN}

La humanidad vive hoy más que una época de cambio y Ecuador no ha estado ajeno a estos procesos. Los últimos años han sido una etapa de profundas transformaciones en todos los campos, especialmente en la Educación Superior que, en la actualidad, tiene dentro de sus objetivos fundamentales dar solución a los problemas y demandas del entorno social, a través de la formación, capacitación y evaluación de los recursos humanos.

La propia sociedad, cada vez más, exige a la universidad certificar los niveles de calidad en el desarrollo de sus procesos sustantivos, y con ello surge, de modo bastante generalizado, la necesidad de las universidades de rendir cuentas a la sociedad de su quehacer. Por ello, la evaluación del desempeño adquiere cada vez mayor relevancia, pues no solo permite conocer la situación actual, lo que falta por hacer, la valoración de la calidad, eficiencia y efectividad de lo logrado, sino intencionar las metas, proyectos institucionales, profesionales y personales, para dominar, así como aplicar con mejor integralidad los nuevos conocimientos, habilidades y competencias requeridos para poder cumplir la misión social.

La evaluación ha de comprenderse como proceso y resultado que estimule la transformación de los profesionales, por medio de un sistema de relaciones e interrelaciones de carácter personal y profesional que garantice y evalúe con integralidad, objetividad, pertinencia y calidad los resultados que se van alcanzando, constituyéndose en un proceso estimulador de la excelencia profesional.

En estos momentos el sistema de Educación Superior de Ecuador es impactado con gran fuerza por los cambios que se producen en él y por aquellos que se derivan de las reglamentaciones existentes en el sector educacional, por ejemplo, el Plan Nacional de Desarrollo del Buen Vivir (2013-2017), la Ley Orgánica de Educación Superior (2010), el Reglamento de Escalafón Docente (2013), por lo cual resulta necesario que el desempeño de los profesores sea eficiente y sea evaluado integralmente como política educativa del país.

El Instituto Superior Tecnológico de Formación Profesional Administrativa y Comercial (ITF), como parte de los institutos tecnológicos superiores en Ecuador, enfrenta la problemática de desarrollar el proceso de mejoramiento del desempeño profesional de los docentes, partiendo de las condiciones y exigencias sociales que garantizan la calidad de dicho proceso, considerando su naturaleza social y la relación que se establece entre la tríada ciencia, tecnología y sociedad.

\section{DESARROLLO}

En el proceso de evaluación de un docente deben participar diferentes agencias o agentes que se relacionan con su desempeño, los cuales influyen de una manera u otra en su actuación profesional y en su evaluación, díganse los estudiantes, la familia, los compañeros de trabajo, los procesos de comunicación social, la institución. A su vez el buen desempeño profesional del docente es vital para el desarrollo social. 
En este sentido, en el modelo educativo de Ecuador han existido algunas incoherencias en la evaluación del desempeño profesional del docente de los institutos superiores tecnológicos, ya que su base teóricaconceptual se sustenta, en gran medida, en el desarrollo de los contenidos teóricos de las diferentes ramas del conocimiento, de acuerdo con una lógica interna de las ciencias, en función de objetivos medibles y observables de tipo conductual, que responden a un paradigma evaluativo centrado en productos.

Lo anteriormente expuesto repercute de forma negativa en la integralidad de la evaluación de los docentes, pues prima el tecnicismo en el manejo de las taxonomías de los objetivos, indicadores y contenidos de la evaluación, donde a veces se desvaloriza la repercusión individual-social que genera este enfoque reduccionista. La evaluación debe ser vista como una cuestión ética más que una cuestión técnica, porque interactúan personas que elaboran juicios de valor sobre una situación determinada en la cual están involucradas otras personas.

La Ley de Educación Superior, expedida en el año 2010, es crucial en este proceso; a partir de su publicación se establece la obligatoriedad de evaluar las instituciones de educación superior, aunque no fue suficientemente explícita para que tuviera el impacto social deseado y se convirtiera en un documento de referencia para que la evaluación del desempeño profesional fuera más ética y humanista.

En correspondencia con lo expuesto, Ecuador estructura el Plan Nacional del Buen Vivir 2013-2017, donde se manifiesta que se debe potenciar el rol de docentes y otros profesionales como actores claves en la construcción del buen vivir en una sociedad cada vez más próspera y sustentable, mejorar la oferta de la formación de docentes y otros profesionales de la educación, en el marco de una educación integral, inclusiva e intercultural.

En tal sentido, la educación en los institutos tecnológicos desempeña un rol esencial para el desarrollo social, donde la calidad y pertinencia de los resultados profesionales de sus maestros y profesores son imprescindibles, lo cual demanda un conjunto de acciones intencionadas benefactoras a la sociedad, entre ellas la de formar estudiantes calificados y competentes, de manera que puedan enfrentar los retos laborales y profesionales en el dominio de la ciencia, la técnica y la tecnología que requiere la sociedad ecuatoriana.

Así, la evaluación constituye un eslabón esencial del proceso docente educativo, la que se interrelaciona dialécticamente con otros componentes, cuyas funciones instructiva, educativa, de diagnóstico, de desarrollo y de control le dan una connotación particular a este proceso como un todo.

La evaluación, en su concepción más general, permite retroalimentar acerca del trabajo realizado y adoptar estrategias de intervención pedagógica individualizadas, en una institución, provincia y país. A partir de sus resultados se genera conciencia social, no sólo acerca de la calidad del aprendizaje de los estudiantes, sino también acerca del desempeño profesional de los docentes y directivos y por consiguiente se generan políticas educativas nacionales.

Es consustancial atender la evaluación del desempeño profesional del docente como un proceso 
esencial dentro de los institutos tecnológicos ecuatorianos, que permita valorar con profundidad los resultados obtenidos por el docente en el proceso docente educativo, en la calidad de sus innovaciones profesionales, las aportaciones y aplicabilidad de los resultados en la práctica educativa en la formación de la personalidad de alumnos, la calidad del aprendizaje, así como los impactos en los propios alumnos, en la institución, en la comunidad y socialmente.

\section{Situación actual del ITF en relación con el proceso de evaluación del desempeño del docente}

Desde 2011 hasta la actualidad, en el ITF se establece legalmente el proceso de evaluación del desempeño de sus docentes atendiendo a la ley de Educación Superior de Ecuador, la cual estipula que los profesores se someterán a una evaluación periódica integral, a partir de la cual se implementa un reglamento escalafonario y se efectúa el proceso de evaluación del desempeño de sus docentes, pero se carece de herramientas metodológicas con una base categorial, donde se enfatice en la integralidad y la ética que debe caracterizar la evaluación de los docentes .

La publicación del Reglamento del Escalafón Docente en el año 2013 constituye otro momento esencial de la investigación, debido a que con esta reglamentación se gana en claridad acerca del perfil que el docente debe cumplir para poder mantenerse y ser promovido en una misma institución.

A pesar de que este reglamento constituye una guía para evaluar a los docentes, todavía los acápites que se establecen para valorar su desempeño quedan enmarcados en aspectos normativos legales, que solo apuntan a elementos externos del quehacer profesional. Resulta positivo que se conciban las actividades y perfiles que deben realizar los docentes en sus funciones cotidianas, aunque procedimentalmente faltan argumentos de cómo actuar para lograr una pertinente evaluación.

El diagnóstico realizado tomó como población una muestra del profesorado, sus directivos y estudiantes a los que se les aplicó el muestreo no probabilístico, cuyo criterio de selección fue aleatorio simple, para conocer cuál es el estado de la evaluación del desempeño en la institución. La muestra se constituyó con 7 directivos de 12, que representa el 84, 4\%. Se investigaron 20 docentes de 26, para un $85 \%$ y 100 de 500 estudiantes de las distintas carreras del instituto, lo que representa el $20 \%$, durante el periodo académico febrero - julio 2015. Se aplicaron distintos instrumentos de investigación como: Guía de observación sobre la evaluación del desempeño del docente en el ITF, entrevistas a directivos y docentes, encuestas a estudiantes, análisis de documentos oficiales. En cuanto a los principales indicadores de la evaluación del desempeño se asumieron los siguientes:

1. Análisis de los procedimientos empleados para la evaluación del desempeño del docente.

2. Valoración del carácter integral y ético durante el proceso evaluativo del docente.

3. Participación de los docentes, de los directivos y los estudiantes en el proceso de evaluación de su desempeño

Los directivos demostraron, en un $55 \%$, desconocimiento de una concepción sólida sobre la forma de 
llevar a cabo el proceso de evaluación del desempeño profesional del docente. El $70 \%$, por su parte, evidenció ciertos conocimientos acerca de algunos indicadores para valorar el desempeño. El $60 \%$ pudo relacionar algunos procedimientos establecidos para llevar a cabo de manera ordenada esta evaluación, El $85 \%$ reconoce que la evaluación docente se hace de manera festinada, carente de un basamento metodológico, lo cual no garantiza la integralidad y el carácter participativo de todos los que, de una manera u otra, tienen que ver con la evaluación de los profesores.

Con respecto a los docentes, el $80 \%$ expresa que la evaluación del desempeño se hace priorizando la presentación de certificaciones que acrediten la consecución de títulos académicos obtenidos, por lo que faltan otras exigencias o indicadores que den cuenta de la integralidad de lo que realizan profesionalmente dentro de la institución.

El $82 \%$ de los profesores plantea que no desempeñan un papel activo en su evaluación, incluso que no se considera la autoevaluación como un procedimiento importante para evaluar su actuación profesional. El $75 \%$ relató situaciones donde se describe falta de ética y rigor en la evaluación hecha por algunos directivos. El 84\% siente insatisfacción al plantear que muchas veces no se evalúa los resultados de su docencia, de sus investigaciones, ni tampoco se valora suficientemente la labor que realizan con sus estudiantes.

Un $45 \%$ reconoce que los directivos valoran lo que aportan con su desempeño a la institución; sin embargo, no se pondera en el momento de evaluarlos. E1 $90 \%$ de los profesores está en desacuerdo con algunos procedimientos y criterios que se utilizan para ser evaluados, por ejemplo, que los estudiantes determinen la evaluación de su desempeño durante el curso académico.

Se deja ver el temor de los docentes a que los resultados de la evaluación del desempeño puedan ser tomados de alguna manera como base de represalias, debido que este proceso puede ser direccionado por personas que carezcan de una formación pertinente, con escasos criterios éticos profesionales, de manera que puedan tergiversar los resultados y no visualizar la evaluación como lo que tiene que ser, un proceso para mejorar su desempeño como docentes.

Existe coincidencia en todos los investigados en cuanto a que la evaluación del desempeño del docente debe contribuir al mejoramiento de la calidad de la educación superior y que es un proceso importante para la mejora institucional, aunque reconocen que aún carecen de una concepción de la evaluación profesoral objetiva, que resulte de la investigación científica. Reconocen que son los directivos los que determinan la proyección evaluativa de sus subordinados y los resultados que se alcanzan se basan en los criterios de los estudiantes.

El $95 \%$ de los docentes y el 100\% de los directivos opina que la evaluación del desempeño debe ser un compromiso de cada una de las personas que forman parte de la institución, con los resultados que esta debe alcanzar. Se reconoce la importancia que puede tener la autoevaluación del propio docente, como parte de un sistema de evaluación del desempeño. Se constata igualmente, la aceptación general de la 
participación de los estudiantes en el proceso de evaluación del desempeño del docente, incluso, por los propios docentes, que reconocen la importancia de tal participación, pero insisten en que no sea lo que determine este proceso.

En cuanto a los estudiantes, se pudo constatar que el $80 \%$ plantea que no se les orienta qué criterios deben tener en cuenta para evaluar justamente a sus docentes, por lo que les resulta difícil evaluarlos. En general, tienen la opinión de que a veces se les pide que evalúen a sus profesores precipitadamente, con poco margen de tiempo para meditar al respecto. El 100\% le concedió gran importancia a la evaluación de sus profesores por los resultados de calidad de la docencia, su participación en la vinculación y lo que aporta a la comunidad estudiantil y a la sociedad.

Entre las limitaciones generales del diagnóstico se aprecia:

Coincidencia entre los sujetos investigados en la necesidad de una participación más activa durante el proceso evaluativo de los docentes, apelando a elevar los niveles de justicia y ética pedagógica en su realización.

Predominan los criterios valorativos de que la evaluación del desempeño profesional en la institución necesita de nuevos fundamentos y procedimientos que estimulen y comprometan a los evaluadores y docentes al cambio educativo.

En la realidad ecuatoriana, los lineamientos de la evaluación del desempeño del profesional aún no se han concebido en un sistema armónico, integral, justo, que permita crear una imagen constructiva de la evaluación, que favorezca al docente y su actuación profesional. Ello implica la necesidad de transitar de un enfoque burocrático a otro más profesional. 


\section{CONCLUSIÓN}

El proceso de la evaluación del desempeño profesional del docente es un proceso complejo, multifactorial y sistémico, que requiere de aportaciones teórico - metodológicas que permitan llenar el vacío epistémico aun existente. El diagnóstico de la situación actual de la evaluación del desempeño profesional del docente en el ITF mostró carencias teórico- metodológicas que generan insatisfacciones en los sujetos involucrados en este proceso. Por lo tanto, es preciso el perfeccionamiento de este proceso evaluativo desde bases científicas en el contexto de los institutos técnicos tecnológicos en la realidad ecuatoriana, a través de nuevas herramientas teórico- metodológicas.

La puesta en marcha de un sistema de evaluación del desempeño docente en las instituciones educativas en el país debería ser implementada cuando haya una general aceptación por parte de la comunidad educativa, fundamentalmente de los docentes. Sin duda, los sistemas de evaluación impuestos que predominan actualmente no logran alcanzar el objetivo de mejorar la calidad de la enseñanza.

Se coincide en integrar la evaluación del sistema educativo y del desempeño profesional en Ecuador, destacando la necesidad de diseñar indicadores basados en un sistema de conceptos que le den justeza y coherencia, así como procedimientos que garanticen su adecuada ejecución. 


\section{REFERENCIAS BIBLIOGRÁFICAS}

Aguerrondo, I. (1997). Calidad de la Educación: ejes para su definición y evaluación. En Revista Interamericana de desarrollo educativo, No.1166, III, OEA.

Álvarez, R. M. (2000). La evaluación del sistema educativo cubano, una experiencia. Revista Educación, No.321.Madrid: España.

Argudín, Y. (2001). La evaluación educativa en la actualidad. Revista Didáctica No.38. Órgano de Centro de Desarrollo Educativo en la Universidad Iberoamericana.

Constitución de la República del Ecuador (2008)

CONEA (2003). El Sistema Nacional de Evaluación y Acreditación de la Educación Superior. Serie documentos técnicos: Hacia el desarrollo de la calidad de la educación Superior ecuatoriana. №1. Nov. Quito. Ecuador.

CONEA (2003). Guía de Auto evaluación con fines de Acreditación. Serie documentos técnicos: Hacia el desarrollo de la calidad de la educación superior

CONESUP (2008). Reglamento de Evaluación del Docente en las Instituciones de Educación Superior. Quito. Ecuador.

Horruitiner, P. (2009). La universidad cubana modelo de formación. Ciudad de La Habana: Editorial Universitaria.

Ley Orgánica de educación Superior del Ecuador (2010)

Plan Nacional de Desarrollo del buen Vivir (2013-2017)

Ruíz, J. (1995). La autoevaluación institucional de Educación Superior. En Revista Iberoamericana de Educación No. 8 mayo - agosto.

Sánchez, A. (2011). Estrategia de evaluación del desempeño del docente universitario ecuatoriano. Tesis en opción al Grado Científico de Doctor en Ciencias de la Educación. Camagüey. Cuba.

Tejedor F, J. y Montero, L. (1998). Indicadores de la calidad docente para la evaluación del profesor universitario. En Revista Española de Pedagogía No. 186, mayo - agosto.

Toranzos, L. (1996). Evaluación y calidad. En Revista Iberoamericana de Educación No. 10, enero abril.

Valdés, Héctor. (1997). Presente y futuro de la meta-evaluación en América Latina.

En Revista

Desafío Escolar, Año 1 volumen 3, noviembre - diciembre, México. 